I.

\title{
Plantæ Hattæ. vel, Materiæ ad Floram Koreanam et Manshuricam.
}

\author{
auctore T. Nakai. \\ Botanicus adjutor in Universitate Imperiale Tokyoense.
}

Accepi nuper nonnulla specimina plantarum ex domino K. HatтA, magister scholæ Agriculturæ et Forticulturæ in Korea. Index collectionum suarum plantarum adhuc mihi concessarum apparuit in meis 'Flora Koreana.' Plantæ in presente opere enumerare adgredior tria genera specimina continent. Plurimam partem vero primum a dom. UEKr. circa Suigen (Korea) lectum, secundum a discipulis suis in variis locis Koreæ discerptum, tertium dom. HATTA ipso serium (ie. in æstate ann. 1910-1911) in regionibus Kirinensis (Manshuria), ubi Illus. Komarov olim diligenter exploravit, lectum est. Alio officio per peragrinationem distrincto, discerpit hic illuc sponte has plantas, neque quæsivit novitates neque investigavit habitates plantarum. Inter eos nonnullas insignas species adsunt et præcipue Neillia est notabillima.

I. Ranunculaceæ.

1) Ranunculus acris L. NakaI F1. Kor. I. 23.

Korea : circa Suigen (Uekr) no. 70.

2) Aquilegia oxysepala Trautv. et Mey. Nakai 1. c. II. 432. Korea : circa Suigen (UEIsI) no 4.

II. Caryophyllaceæ.

3) Krascheninnikowia heterophylla MiQ. in Ans. Mus. Bot. Lugd. Bat. III. 187. Palib. Consp. F1. Kor. I. 41.

Korea : circa Suigen (UEKI). 
III. Guttiferæ.

4) Hypericum Yabei Léveillé et Vaniot. Nakai Fl. Kor. I. 97.

Korea : circa Suigen (UEKI) no. 60.

IV. Tiliaceæ.

5) Tilia amurensis Kom. F1. Mansh. III. 24. NaKaI 1. c. I. 105 .

Manshuria : Chang-Kwan-Tsee-Ring (張廣哩嶺). Junio 16. 1911. (K. Hatta) no. 17.

6) Tilia mandshurica Rupr. et Maxim. Kom. F1. Mansh. III. 28. NAKAI I. 106. II. 454.

Manshuria: Chang-Kwan-Tsee-Ring. Junio 16. 1911. (K. Hatтa) no. 19.

\section{Rutaceæ.}

7) Phellodendron amurense RUpr. Kom. II. 668. NAKAI I. 117.

Manshuria: Chang-Kwan-Tsee-Ring, Junio 16. 1911 (K. HatTa) no. 20.

VI. Rhamnaceæ.

8) Rhamnus daurica Pall. Kom. III. 9. NaKaI I. 125. II. 460 .

Manshuria: Chang-Kwan-Tsee-Ring, Junio 16. 1911 (K. НАTTA) no. 10.

VII. Aceraceæ.

9) Acer mandshuricum Maxim. Kom. II. 727. NAKAI I. 132 .

Manshuria: Chang-Kwan-Tsee-Ring, Junio 16. 1911 (K. HATTA) no. 11.

VIII. Leguminosæ.

10) Cassia mimosoides L. var. dimidiata Hook، fil. NAKai I. 142.

Korea : circa Suigen (Ueki) no. 8. 
11) Lespedeza cyrtobotrya Mig. Prol. Fl. Jap. 236. NAKAI I. 155.

Korea : sine loco speciali (a discipulis). d. g. e.

12) Lespedeza striata Hook. et Arv. Bot. Beech. Voy. 262. Folia elliptica nunquam obovato-elliptica, sed junior planta haud raro folia obovata agit. Stipula quam var. stipulacea Makino. (L. stipulacea Maxim.) angustior, ramusque densius congestus. Specimina exsiccata inter sese simillimum fit, sed viva primo obtutu distinguere possimus.

Korea : circa Suigen (UEKr).

Planta nova ad Floram Koreanam.

13) Lespedeza bicolor Turcz. var. intermedia Maxim. in Act. Hort. Petrop. II. 356. Nakai I. 156.

Korea : sine loco speciali (a discipulo) $\mathrm{f}$.

var. Sieboldii Maxim. 1. c. Nakai I. 156.

Korea : sine loco speciali (a discipulo) h.

14) Cladrastis amurensis (Rupr. et Maxim.) Benth. Gen. P1. I. 554. NakaI I. 167.

Manshuria : Chang-Kwan-Tsee-Ring. Junio 16. 1911. no. 10. (K. НатTA).

\section{Rosaceæ.}

15) Neillia Uekii sp. nov.

Specimen mancum, tantum fructus et folia portat, sed evidenter species Neilliæ nova est.

Ramus gracilis, subangulato-striatus, modo alliis Neilliis similis, annotinus incanus, hornotinus squamis imbricatis suffultus, pubescens. Folia petiolata et stipulata. Petiolus arcuatus 3-6 $\mathrm{mm}$. longus pubescens. Stipulæ caducæ. Lamina foliorum anbitu ovata v. late-ovata v. oblongo-ovata, forma et serris Neillix sinense ommino similis, supra glaberrima, subtus ad nervos pilosula. Racemus ad apicem rami hornotini terminalis, fructiferum 4-6 cm. longus; pars fructifera 2.5-4.3 $\mathrm{cm}$. longa; rhachis pilosula. Pedicellus $2.5-3 \mathrm{~mm}$. longus parce glanduloso-hispidus. Calyx ovatus dense glandulosoaculeatus, ciliis obsoletis intermixtus. Glandula $1.5-2 \mathrm{~mm}$. longa. Tubus calycis $6 \mathrm{~mm}$. longus, 10 nervis. Dentes calycis 
5 elongato-triangulati $3 \mathrm{~mm}$. longi, conniventes sed apice parce reflexi. Carpellum 1, ovatum apice acutum ventrali-dehiscense biseminibus. Styli $3 \mathrm{~mm}$. longi persistentes. Semina horizontali ornata. Semen obovatum lucidum albuminatum. Testa coriacea, ventrali unijugalis. Embryo erecta. Cotyledones laterali positi.

Korea : sine loco speciali (a discipulo) p.

Species inter $N$. thyrsiflora et $N$. sinensis interstat. Glandula calycis priori similis et forma foliorum posteriori appropinquit.

Genus Neillia est incola Asiæ. Sex species adhuc notæ sunt. Omnes in regionibus Himalayæ et Chinæ indigenæ sunt, ita ex regionibus Manshuria-Koreanis nulla nota erat. Inventio hujus species est res notabillima, et distributionem hujus generis ad has regiones amplificat.

16) Cratægus pinnatifida Bunge Enum. pl. Chin. bor. n. 157. Kom. II. 466. NaKaI I. 179.

Manshuria: Chang-Kwan-Tsee-Ring. Junio. 16. 1911 no. 16. (K. НаtтA).

17) Rubus pungens Camb. Nakai I. 187.

Korea : circa Suigen (UEKI).

18) Pirus Calleryana Desne. Nakai I. 181.

Korea : circa Suigen (UEKI).

19) Pirus baccata L. var. sibirica Maxim. in Mel. Biol. IX. 166. Kom. Fl. Mansh. II. 475. Nakai F1. Kor. I. 181.

Manshuria: Ka-y-pu-hu(卡一巴河)Junio 13. 1911 (K. HatTA)

20) Geum strictum Ait. Kom. II. 517. Nakai I. 200.

Manshuria：Wang-Da-Hu-Tsu（横道河子）Junio 30. 1911 no 1. et no. 5 (K. Hatta).

21) Potentilla Kleiniana Wight et Arn. Nakai I. 198.

Korea : circa Suigen (Ueki) no. 7.

X. Saxifragaceæ.

22) Philadelphus coronarius L. $\gamma$. Satsumi (Sieb.) Maxim. NAKAI I. 221.

Manshuria: Wang-Da-Hu-Tsu. Junio 30. 1911 no. 3. (K. HATTA).

23) Deutzia parviflora Bunge Kom. II. 431. NaKaI I. 222. 
Manshuria: Wang-Da-Hu-Tsu, Junio 30. 1911 no. 2. (K. Hatta).

XI. Oenotheraceæ.

24) Ludwigia prostrata Roxb. NAKaI I. 239.

Korea : circa Suigen (UEKI). no. 81.

XII. Umbelliferæ.

25) Sium Ninsi L. Nakai I. 260.

Korea : circa Suigen (UekI) no. 73.

XIII. Araliaceæ.

26) Eleutherococcus senticosus Maxim. Kom. F1. Mansh. III. 119.

Manshuria: Chang-Kwan-Tsee-Ring. Junio 16. 1911. no. 14. (K. HatTa).

XIV. Caprifoliaceæ.

27) Viburnum Opulus L. var. Sargentii Takeda. NakaI II. 495 .

Manshuria: Chang-Kwan-Tsee-Ring. Junio 16. 1911 no. 9. (K. Hatta).

28) Lonicera Ruprechtiana REgeL Gartenfl. XIX. 68. t. 645. Kom. F1. Mansh. III. 523.

Korea : sine loco speciali (a discipulo). $\mathrm{j}$.

Planta nova ad Floram Koreanam.

XV. Valcrianaceæ.

29) Valeriana officinalis L. NakaI I. 302. II. 504.

Manshuria : Wang-Da-Hu-Tsu. Junio $30 \quad 1911$ no 4. (K. HatTA).

\section{Compositæ.}

30) Bidens tripartita L. NaKaI II. 20.

Korea : circa Suigen (UeKr) no. 19.

31) Saussurea affinis Spreng. NakaI II. 42.

Korea : circa Suigen, no. 21. (UEKI).

32) Centipeda minima (L.) O. Kuntze Nakai II. 26.

Korea : circa Suigen, no 61. (UEKI). 
33) Cirsium Maackii Maxim. NakaI II. 47.

Korea : circa Suigen no. 74. (UекI).

34) Erigeron canadensis L. NaKaI II. 12.

Korea : circa Suigen, no. 80. (UeKr).

35) Sonchus oleraceus L. NaKaI II. 53.

Korea : circa Suigen, no. 66. (UEKr).

36) Lactuca versicolor (Frscher) Sch.-Bip. NakaI II. 56.

Korea : circa Suigen no. 29. (UEKI).

37) Lactuca Bungeana NaKaI II. 56.

Korea : sirca Suigen. no 56 et 82 . (UEKI).

38) Hieracium hololerion Maxim. Nakai II. 58.

Korea : circa Suigen. no. 2. (Ueki).

XVII. Campanulaceæ.

39) Adenophora verticillata Fischer NAKaI II. 65.

Korea . circa Suigen. no 51. (UEkr).

XVIII. Primulaceæ.

40) Androsace saxifragæfolia Bunge Nakai II. 79.

Korea : circa Suigen (UEkI).

XIX. Styracaceæ.

41) Symplocos cratægoides Ham. Nakai II. 85.

Korea : sine loco speciali (a discipulo) q.

XX. Polemoniaceæ.

42) Polemonium cæruleum L. NAKAI II. 101.

Korea : circa Suigen. no. 3. (Uekr).

XXI. Borrhaginaceæ.

43) Brachybotrys paridiformis Maxim. NakaI II. 102.

Korea : circa Suigen no. 5. (UEkı).

XXII. Solanaceæ.

44) Physalis minima L. NakaI II. 114.

Korea : circa Suigen, no. 52. (UEKI).

XXIII. Scrophulariaceæ.

45) Mazus japonicus (Thunb.) O. Kuntze NaKaI II. 119.

Korea : circa Suigen, no. 11. (UEKI). 
XXIV. Labiatæ.

46) Stachys aspera Michx. var. chinensis Maxim. forma glabrata NAKAI II. 147.

Korea : circa Suigen no. 57. (UEkr)

47) Salvia plebia R. BR. NAKar II. 141.

Korea : circa Suigen no. 63. (UeKI).

48) Meehania urticifolia Kom. NaKaI. II. 151.

Korea : circa Suigen no. 2. (UEKI).

49) Mosla grosse-serrata Maxim. NakaI II. 145.

Korea : circa Suigen. no. 26. (Uerr).

XXV. Polygonaceæ.

50) Polygonum Persicaria L. Nakai II. 167.

Korea : circa Suigen. no. 34. (Uekr).

XXVI. Santalaceæ.

51) Thesium chinense Turcz. Nakai II. 180.

Korea; circa Suigen no. 14. (UEKI).

XXVII. Euphorbiaceæ.

52) Phyllanthus ussuriensis Rupr. et Maxim. NakaI II. 182.

Korea : circa Suigen. no. 25 et 30 (UEKI).

XXVIII. Ulmaceæ.

53) Ulmus montana With. var. laciniata TraUtv. NakaI II. 190.

Manshuria: Chang-Kwan-Tsee-Ring. Junio 16. 1911. no. 13. (K. Hatta).

54) Celtis sinensis Pers. Nakai II. 192.

Korea : sine loco speciali (a discipulo) $n$.

55) Celtis Bungeana PL. Nakai II. 192.

Korea : sine loco speciali (a discipulo) o.

XXIX. Betulacaceæ.

56) Betula daurica Pall. Nakai II. 203.

Korea : circa Suigen (UEKI) sine loco speciali (a discipulo) c.

57) Carpinus cordata BL. NaKaI II. 205. 
Manshuria: Chang-Kwan-Tsee-Ring. Junio 16. 1911. no. 12. (K. Hatta.)

XXX. Salicaceæ.

58) Salix vagans ANders, var. cinerascens ANders. NaKaI II. 213.

Manshuria: Chang-Kwan-Tsee-Ring. Junio 16. 1911 no. 21. (K. Hatta).

59) Salix purpurea L. NaKaI II. 215.

Korea : sine loco speciali (a discipulo) k.

60) Salix cinerea L. NAKAI II. 213.

Korea : sine loco speciali (a discipulis) 1. et s.

61) Populus tremula L. NaKai II. 211.

Korea : sine loco speciali (a discipulo) $\mathrm{m}$.

XXXI. Iridaceæ.

62) Iris ensata Thunb. var. chinensis Maxim. NakaI II. 231. Manshuria. Tong-Kong-Kwi-Tsu (通南滗子). Junio 14. 1911 (K. HatTA).

63) Iris sibirica L. var. orientalis Thunb. NAKaI II. 233.

Korea : circa Suigen no. 67. (UEKI).

XXXII. Liliaceæ.

64) Trillium obovatum PUrsh. NakaI II. 240.

Korea : circa Suigen no. 8. (Uekr).

65) Allium tenuissimum L. NakaI II. 259.

Korea : circa Suigen no. 83. (UEKI).

XXXIII. Juncaceæ.

66) Luzula campestris L. var. capitata MIQ. NAKAI II. 268. Korea : circa Suigen (UEKI).

XXXIV. Cyperaceæ.

67) Cyperus Iria L. Nakai 1. c. II. 288.

Korea : circa Suigen no. 3 (UEKI).

68) Fimbristylis diphylla VAHL. NaKaI II, 291.

Korea : circa Suigen no. 28. (UEKI).

69) Fimbristylis miliacea VAHL. NAKAI II. 290.

Korea : circa Suigen no. 53. (UEKI). 
70) Bulbostylis capillaris L. var. trifida (KunTH) C. B. Clarke Nakai II. 295.

Korea : circa Suigen no. 62. et 78. (UEKI).

71) Carex pumila Thunb. NakaI II. 333.

Korea : circa Suigen no. 75. (UEKI).

72) Carex Arnelli Christ. Nakai II. 325.

Korea : circa Suigen no. 6. (UekI).

73) Carex nubigena Don. v. ablata (BootT.) KüK. NAKAI II. 305 .

Korea : circa Suigen no. 49. (UEKI).

74) Carex neurocarpa Maxim. Nakai II. 289.

Korea : circa Suigen no. 57. (UEKr).

XXXV. Graminæ.

75) Rottbœlia compressa L. $\delta$. japonica Hackel. NakaI II. 341 .

Korea : circa Suigen no. 68. (UEKI).

76) Arthraxon ciliaris Beauv. $\alpha$. genuinus Hackel. NATAI II. 343.

Korea : circa Suigen no. 79. (UEKI).

77) Andropogon Nardus L. var. Gœringii HackeL NAKaI II. 343.

Korea : circa Suigen no. 15. (UEKI).

78) Andropogon brevifolius Sw. NakaI II. 343.

Korea : circa Suigen no. 23. (UeKI)

79) Panicum Crus-Galli L. var. genuinum HACKeL NAKAI II. 347 .

Korea : circa Suigen no. 5. et 32 (Uekr). var. frumentaceum Hook. fil. Nakai 1. c. Korea : circa Suigen no. 27,48 et 76 . (UEKI). 80) Panicum acroantha Steud. Nakai II. 346. Korea : circa Suigen no. 64. (UEKI)

81) Panicum indicum L. NaKaI II. 347. Korea : circa Suigen no. 13 et 72. (UEKI). 82) Setaria viridis Beauv. NakaI II. 350. Korea : circa Suigen no.' 9. et 77. (UeкI). 
83) Phalaris arundinacea L. $\alpha$. genuina Hackel. NakaI II. 352 .

Korea : circa Suigen no. 58. (UEKI).

84) Cinna pendula Trin. NaKai II. 356.

Manshuria: Chang-Kwan-Tsee-Ring. Junio 16. 1911 no. 7. (K. Hatta).

85) Agrostis perennans Tuck. Nakai II. 359.

Korea : circa Suigen no. 69. (UEKI).

86) Trisetum flavescens Beauv. var. macrantha Hackel. NAKaI II. 360.

Korea ; circa Suigen no. 1. et 71. (Ueki).

87) Eragrostis pilosa Beauv. Nakai II. 366.

Korea : circa Suigen (UEkI).

88) Eragrostis ferruginea Trin. NaKaI II. 365.

Korea : circa Suigen no. 24. (Ueki).

89) Poa strictula Steud. Nakai II. 371.

Korea : circa Suigen no. 65. (UEKI).

90) Bromus ciliatus L. NakaI II. 374.

Korea : circa Suigen no. 38 (UeKI).

91) Agropyrum ciliare Franch. Nakai II. 375.

Korea : circa Suigen no. 12, 16. et 17. (UEKI).

92) Arundinaria japonica Sieb. et Zucc. NaKai II. 377.

Korea：Kyöng-geui : Daisonri (京畿道, 道律郡, 大坡面, 大村 里). (UEKI).

XXXVI. Pinaceæ.

93) Abies nephrolepis Maxim. Narai II. 381.

Manshuria: Chang-Kwan-Tsee-Ring. Junio 16. 1911 (K. HATTA).

XXXVII. Equisetaceæ.

94) Equisetum hiemale L. NAKAI II. 421.

Korea : circa Suigen no. 22. (UEKr). 